Background: Epidemiological studies assessing rates of postnatal depression in relation to levels of fish consumption and reduced levels of omega- 3 in the perinatal period indicate a possible link between lowered long-chain omega- 3 fatty acids and perinatal depression.

Aim: To assess whether omega-3 fatty acid treatment is superior to placebo in the treatment of perinatal depression.

Methods: In a double-blind, randomized, placebocontrolled treatment trial, we recruited women from third trimester to 6 months postpartum with a diagnosis of major depression. Treatment was for 6 weeks with either $6 \mathrm{~g}$ of fish oil or placebo. Weekly followup was carried out by a psychiatrist and depression rating scales recorded. Fatty acid analysis was conducted on blood samples collected at baseline and posttreatment trial.

Results: There was significant improvement in depression scores for the treatment group during the trial. However, a repeated-measures ANOVA showed no statistically significant difference between treatment and placebo groups using intention-to-treat analysis. Trend for efficacy differentiation were noted when nonresponders and rapid remitters were excluded.

Conclusions: These results are likely to be affected by a strong placebo response, which is a common problem in clinical trials for the treatment of depression. Further study is needed in this area because treatment options are limited for perinatal depression.

\section{Understanding how young people cope with distress: the development of a mobile phone momentary sampling program (Mobile_TYPE)}

\section{S Reid', G Patton', L Sanci'2, S Kauer'}

'Murdoch Childrens Research Institute; and 'Department of General Practice, University of Melbourne, Melbourne, Australia

Background: Young people report that they typically respond to distress by talking to their friends, thinking and distracting themselves. By using retrospective questionnaires, research has examined what young people say but not what they actually do when distressed and what the impact of these responses are prospectively. The aim of this study was to design a mobile phone momentary sampling program that prospectively tracks the everyday mood, stresses and coping strategies of young people as they happen.

Design: A momentary sampling program was designed and translated into java-based language for mobile phones. The program ran for 7 days, administering four random samples per day. Participants were prompted to report current activity, companions, mood, response to low mood, recent stressful experiences, and alcohol and cannabis use. The program was reviewed by 11 high school students in focus groups, and 18 students completed 7 days of mobile monitoring.

Results: Engagement with the Mobile_TYPE program was high with a majority of students $(82 \%)$ completing at least $75 \%$ of the entries. The most common responses to feeling distressed were do nothing (14.6\%), eating $(11.4 \%)$, sleeping (10.8\%) and studying (7.5\%). About $88 \%$ of active responses to distress lead to a later report of feeling good or better. The responses associated with a later report of not feeling better were worrying, doing nothing, crying, relaxing, eating, studying, pretending to be okay and shopping.

Conclusion: The Mobile_TYPE program captured a range of detailed and interesting qualitative, quantitative and prospective data about young people's everyday mood, stresses, responses and general functioning.

\section{Using ketamine to model thought disorder in schizophrenia}

\section{S Rossell', V Curran², C Morgan² \\ 'Mental Health Research Institute, Melbourne, Australia; and 2University College London, London, United Kingdom}

Background: Ketamine is used acutely as a model of schizophrenia. It has been suggested that chronic ketamine use may also mimic aspects of this disorder, in particular cognitive function. Semantic processing deficits are considered to be central to cognitive impairments in schizophrenia and are related to thought disorder. This study aimed to characterize semantic impairments following both acute and chronic ketamine. Methods: We examined the acute effects of ketamine using a double-blind, placebo-controlled, independent groups design with 48 volunteers examining the effects of two doses of ketamine $(100 \mathrm{ng} / \mathrm{ml}$ and $200 \mathrm{ng} / \mathrm{ml})$. The chronic effects of ketamine were explored with 32 volunteers, 16 regular ketamine users and 16 matched polydrug controls. Semantic processing was examined using a lexical-decision semantic priming task with a frequency (high and low) and stimulus onset asynchrony (SOA; short $-200 \mathrm{~ms}$, long $-750 \mathrm{~ms}$ ) manipulation. Schizophrenic and dissociative symptoms were also examined.

Results: Acute ketamine produced a dose-dependent reduction in priming (hypopriming) and increased schizophrenic thought disorder. Ketamine users showed impaired priming for low-frequency words at the long SOA compared with polydrug controls, and there was some evidence of increased priming for high-frequency words. Ketamine users did not differ 